\title{
Invexidade Generalizada e Soluções Fracamente Eficientes de Problemas de Otimização Vetorial entre Espaços de Banach
}

L.B. dos SANTOS ${ }^{1}$, Universidade Estadual de Campinas. Instituto de Matemática

e Computação Científica, Depto. de Matemática Aplicada.

R. OSUNA-GÓMEZ2, Universidad de Sevilla. Facultad de Matemáticas, Depto. de Estadística e Investigación Operativa.

M.A. ROJAS-MEDAR, Universidade Estadual de Campinas. Instituto de Matemática e Computação Científica, Depto. de Matemática Aplicada.

A.R. LIZANA ${ }^{4}$, Universidad de Sevilla. Facultad de Matemáticas, Depto. de Estadística e Investigación Operativa.

Resumo. Neste trabalho, introduzimos a noção de ponto crítico vetorial e de ponto crítico de Kuhn-Tucker para uma certa classe de problemas de otimização vetorial entre espaços de Banach. Através destas noções, obtivemos uma caracterização para as soluções fracamente eficiente de tais problemas.

\section{Introdução}

Em otimização escalar, as condições de Kuhn-Tucker são suficientes para a otimalidade quando todas as funções envolvidas são convexas. Atualmente, consideráveis progressos têm sido obtidos com o intuito de enfraquecer as hipóteses de convexidade de maneira a ampliar a classe de problemas que verificam a suficiência das condições de Kuhn-Tucker.

Uma importante contribuição neste sentido foi dada por Hanson em [6]. Para este fim, considerou as funções invexas. Para tais funções, as clássicas condições de Kuhn-Tucker são suficientes para garantir otimalidade global. Mais tarde, Martin [10] observou que em problemas sem restrições, a invexidade é condição necessária e suficiente para garantir a otimalidade global. Assim, surge a seguinte questão: Qual

\footnotetext{
${ }^{1}$ Aluna de Doutorado em Matemática Aplicada, parcialmente financiada por CNPq e CAPES. E-mail:lucelina@ime.unicamp.br.

${ }^{2}$ E-mail: rafaela@us.es

${ }^{3}$ Este autor é financiado por CNPq-Brazil, processo no. 301354/03-0. E-mail: marko@ime.unicamp.br

${ }^{4}$ E-mail: rufian@us.es
} 
é a maior classe de funções para a qual as condições de Kuhn-Tucker são necessárias e suficientes para garantir a otimalidade global? A resposta a esta questão foi dada por Martin em [10] para problemas escalares.

Consideremos o seguinte problema escalar irrestrito:

$$
\left\{\begin{array}{ll}
\text { Minimizar } & \theta(x) \\
\text { sujeito a } & x \in S \subseteq \mathbb{R}^{n}
\end{array},\right.
$$

onde $\theta(x)$ é uma função escalar e $S \subseteq \mathbb{R}^{n}$.

Recordemos que $\bar{x}$ é um ponto estacionário se $\nabla \theta(\bar{x})=0$ e o problema de otimização com restrições

$$
\left\{\begin{array}{ll}
\text { Minimizar } & \theta(x) \\
\text { sujeito a } & -g_{j}(x) \leq 0 j=1, \ldots, m \\
& x \in S \subseteq \mathbb{R}^{n}
\end{array},\right.
$$

onde $\theta(x)$ é uma função escalar e $g=\left(g_{1}, \ldots, g_{m}\right): \mathbb{R}^{n} \rightarrow \mathbb{R}^{m}$ é uma função vetorial, ambas diferenciáveis no conjunto aberto $S \subseteq \mathbb{R}^{n}$.

Também, lembramos que $(\bar{x}, \bar{u}) \in S \times \mathbb{R}^{m}$ é um ponto estacionário Kuhn-Tucker [9], se

$$
\begin{aligned}
\nabla \theta(\bar{x})+\bar{u}^{T} \nabla g(\bar{x}) & =0, \\
\bar{u}^{T} g(\bar{x}) & =0, \\
\bar{u} & \geq 0 .
\end{aligned}
$$

Além disso, uma função diferenciável $\theta: S \subseteq \mathbb{R}^{n} \rightarrow \mathbb{R}$ é chamada invexa em $\bar{x}$ se existe uma função vetorial $\eta: S \times S \longrightarrow \mathbb{R}^{n}$ tal que

$$
\theta(x)-\theta(\bar{x}) \geq\langle\nabla \theta(\bar{x}), \eta(x, \bar{x})\rangle
$$

e dizemos que é invexa em $S_{0} \subseteq S$, se é invexa em cada ponto $\bar{x} \in S_{0}$.

Definição 1.1. (Hanson e Mond, [6]) A função $\theta: S \subseteq \mathbb{R}^{n} \rightarrow \mathbb{R}$ é chamada pseudoinvexa em $u \in S$ se existe uma função $\eta: S \times S \rightarrow \mathbb{R}^{n}$ tal que

$$
\langle\nabla \theta(u), \eta(x, u)\rangle \geq 0 \Longrightarrow \theta(x) \geq \theta(u), \forall x \in S .
$$

A função $\theta$ é chamada pseudoinvexa em $S$ se $\theta$ é pseudoinvexa em cada ponto de $S$.

Observamos que, para o caso escalar, os conceitos de invexidade e de pseudoinvexidade coincidem. Em [10], Martin provou o seguinte resultado para problemas sem restrições:

Teorema 1.1. A função $\theta$ é invexa em $S$ se, e somente se, cada ponto estacionário é um mínimo global de $\theta$ em $S$.

Entretanto, para problemas com restrições, a invexidade apenas garante a suficiência para a otimalidade. Martin [10] define uma classe de problemas para os quais cada ponto crítico Kuhn-Tucker é ótimo global. Desta maneira, ele obtém 
uma caracterização completa das soluções para o problema com restrições, dada em termos dos pontos críticos.

Lembramos que o problema (CP) é KT- invexo em $S$ se existe uma função vetorial $\eta: S \times S \longrightarrow \mathbb{R}^{n}$ tal que, $\forall x_{1}, x_{2} \in S \operatorname{com} g\left(x_{1}\right) \leq 0$ e $g\left(x_{2}\right) \leq 0$, então

$$
\begin{gathered}
\theta\left(x_{1}\right)-\theta\left(x_{2}\right) \geq\left\langle\nabla \theta\left(x_{2}\right), \eta\left(x_{1}, x_{2}\right)\right\rangle \\
-\left\langle\nabla g_{j}\left(x_{2}\right), \eta\left(x_{1}, x_{2}\right)\right\rangle \geq 0, \forall j \in I\left(x_{2}\right),
\end{gathered}
$$

onde $I\left(x_{2}\right):=\left\{j \in\{1, \ldots, m\}: g_{j}\left(x_{2}\right)=0\right\}$ é o conjunto dos índices das restrições ativas em $x_{2}$. O resultado obtido por Martin em [10] é:

Teorema 1.2. Todo ponto estacionário Kuhn-Tucker para $(C P)$ é um mínimo global de $(C P)$ se, e somente se, $(C P)$ é KT-invexo.

Antes de formularmos os problemas de otimização vetorial que serão tratados neste trabalho, relembramos algumas conceitos preliminares.

Dado um conjunto arbitrário $E$, uma relação binária em $E$ é, por definição, um subconjunto $B$ de $E \times E$, ou seja um elemento $x \in E$ está relacionado com $y \in E$ se $(x, y) \in B$.

Seja $B$ uma relação binária em $E$. Dizemos que $B$ é

1. Reflexiva - se $(x, x) \in B$ para cada $x \in E$. (caso contrário, é chamada irreflexiva);

2. Simétrica - se $(x, y) \in B \Rightarrow(y, x) \in B, \forall x, y \in E$ (caso contrário, é assimétrica);

3. Transitiva - se $(x, y),(y, z) \in B \Rightarrow(x, z) \in B, \forall x, y, z \in E$;

4. Completa - se $\forall x, y \in E, x \neq y$ se tem $(x, y) \in B$ ou $(y, x) \in B$;

5. Linear (no caso em que $E$ é um espaço vetorial) - se $(x, y) \in B$ implica $(t x+z, t y+z) \in B, \forall x, y, z \in E, t>0$.

Uma relação binária é dita ser uma ordem parcial se é reflexiva e transitiva. Se, além disso, for completa é dita ser uma ordem total

É bem conhecido (veja [8]) que se $B$ é uma ordem parcial linear (definida em um espaço vetorial), então o conjunto

$$
C=\{x \in E:(x, 0) \in B\}
$$

é um cone convexo. Se, além disso, $B$ for simétrica, então $C$ é um cone ponteado (isto é, $C \cap(-C)=0$ ). Reciprocamente, cada cone convexo $C$ em $E$ fornece uma relação binária

$$
B_{C}=\{(x, y) \in E \times E: x-y \in C\},
$$

a qual é reflexiva, transitiva e linear.

Por este motivo, consideraremos neste trabalho, apenas relações binárias definidas por cones convexos. 
No que segue, utilizaremos as seguintes notações: Seja, $F$ um espaço de Banach, $C \subset F$ um cone convexo, fechado, ponteado (i.e., $C \cap(-C)=\{0\}$ ), distinto de $F$ e com interior não vazio,

$$
\begin{aligned}
& x \varliminf_{C} y \quad \Leftrightarrow y-x \in C, \\
& x \leq_{C} y \Leftrightarrow y-x \in C \backslash\{0\}, \\
& x<_{C} y \quad \Leftrightarrow y-x \in \operatorname{int} C .
\end{aligned}
$$

Desta maneira, o cone convexo $C \subset F$ caracteriza a preferência do decisor (por exemplo, $y$ é preferível a $x$ se $x \leqq_{C} y \Leftrightarrow y-x \in C$ ).

Lembramos que $h: E \longrightarrow F$ é Fréchet diferenciável no ponto $x$ (veja [1]) (por brevidade, diferenciável no ponto $x$ ) se existe um operador linear e contínuo $D h(x): E \longrightarrow F$ tal que

$$
\lim _{z \rightarrow 0} \frac{\|h(x+z)-h(x)-D h(x) z\|_{F}}{\|z\|_{E}}=0 .
$$

A função $h$ é dita ser diferenciável em $S \subseteq E$ se $h$ é diferenciável em cada ponto de $S$. Sejam $E$ e $F$ dois espaços de Banach e $f: E \longrightarrow F$ e $g: E \longrightarrow G$ duas funções diferenciáveis sobre o conjunto aberto e não vazio $E, S \subset E$, e assumimos que $F$ é parcialmente ordenado pelo cone convexo fechado, ponteado e com interior não vazio $C \subset F(\operatorname{com} C \neq E)$, e $K \subset G$ é um cone convexo fechado e distinto de $G$. Os problemas que serão considerados são:

(1) Sem restrições:

$$
\left\{\begin{array}{ll}
\text { Minimizar } & f(x) \\
\text { sujeito a } & x \in S \subseteq E
\end{array} .\right.
$$

(2) Com restrições:

$$
\left\{\begin{array}{ll}
\text { Minimizar } & f(x) \\
\text { sujeito a } & -g(x) \in K \\
& x \in S \subseteq E
\end{array} .\right.
$$

Observamos que para (VOP) o conjunto factível é $S$ e para (CVOP) é

$$
\mathcal{F}:=\{x \in S:-g(x) \in K\} .
$$

Os seguintes conceitos são conhecidos:

Definição 1.2. O ponto factível $\bar{x} \in S$ é chamado solução eficiente se não existe $x$ factivel tal que $f(\bar{x})-f(x) \in C \backslash\{0\}$ (ou, equivalentemente $\bar{x}$ factivel é eficiente se não existe $x$ factível tal que $\left.f(x) \leq_{C} f(\bar{x})\right)$.

Definição 1.3. O ponto factível $\bar{x} \in S$ é chamado solução fracamente eficiente se não existe $x$ factível tal que $f(\bar{x})-f(x) \in$ int $C$ (ou equivalentemente, $\bar{x}$ é uma solução fracamente eficiente se não existe $x$ factivel tal que $\left.f(x)<_{C} f(\bar{x})\right)$. 
Denotaremos $F^{*}$ o dual topológico de $F$, e $\langle\cdot, \cdot\rangle$ a dualidade canônica entre $F^{*}$ e $F$. Dado um cone convexo $C \subset F$ define-se o cone dual de $C$,

$$
C^{*}:=\left\{\xi \in F^{*}:\langle\xi, x\rangle \geq 0, \forall x \in C\right\} .
$$

Vale notar que, nos últimos anos, têm aparecido novos tipos de funções que pretendem generalizar as que citamos anteriormente. São o caso, por exemplo, das funções F-convexas, $\rho$-convexas e (F, $\rho$ )-convexas (veja [5], [7], [11]). Entretanto, embora seja verdade que estas funções generalizem as que tratamos neste trabalho, pode-se demonstrar (e isto é feito em detalhes em [13]) estas não conduzem a nada novo sobre a otimalidade, porque para provar alguma condição de otimalidade é necessário impor certas hipóteses adicionais, de tal forma que recaem em algum dos tipos tratados neste trabalho.

O trabalho tem a seguinte estrutura: na Seção 2, estudamos o problema sem restrições; definimos funções pseudoinvexas e provaremos que para este problema, $f$ é pseudoinvexa se e somente se ser ponto crítico vetorial é condição necessária e suficiente para a eficiência fraca. Na Seção 3, consideraremos o problema com restrições e definimos problemas KT-invexos; provamos que esta classe de problemas tem a propriedade de que ser ponto crítico Kuhn-Tucker é condição necessária e suficiente para a eficiência fraca.

\section{Condições Necessárias e Suficientes de Otima- lidade para o Problema Irrestrito}

Sejam $E$ e $F$ dois espaços de Banach, $C \subset F$ um cone convexo fechado com interior não vazio e diferente do espaço todo $F$. Seja $S$ um subconjunto aberto e não vazio de $E$ e $f: E \longrightarrow F$ uma função diferenciável em $S$.

Definição 2.1. $\bar{x} \in S$ é um ponto crítico vetorial de (VOP) se existe $\lambda^{*} \in$ $C^{*} \backslash\{0\}$ tal que $\lambda^{*} \circ \operatorname{Df}(\bar{x})=0$.

O seguinte resultado é provado em [2].

Teorema 2.1. Se $\bar{x} \in S$ é uma solução fracamente eficiente de (VOP), então $\bar{x}$ é um ponto crítico vetorial.

Definição 2.2. Seja $f: S \subseteq E \longrightarrow F$ uma função diferenciável no conjunto aberto $S$. Dizemos que $f$ é pseudoinvexa em $S$ com respeito a $\eta$, se existe uma função vetorial $\eta: S \times S \longrightarrow E$ tal que

$$
x_{1}, x_{2} \in S \text { e } f\left(x_{1}\right)-f\left(x_{2}\right)<_{C} 0 \Rightarrow D f\left(x_{2}\right) \eta\left(x_{1}, x_{2}\right)<_{C} 0
$$

(onde $D f\left(x_{2}\right) \eta\left(x_{1}, x_{2}\right)$ denota o valor da função $D f\left(x_{2}\right) \in \mathcal{L}(E, F)$ aplicada no vetor $\eta\left(x_{1}, x_{2}\right) \in E$ e $\mathcal{L}(E, F)$ é o conjunto dos operadores lineares e contínuos de E em $F)$.

Para provar que pontos críticos coincidem com as soluções fracamente eficientes do problema (VOP) quando a função $f$ é pseudoinvexa, necessitaremos do seguinte resultado, que pode ser encontrado em [3], 
Lema 2.1. Sejam $F$ um espaço de Banach, $C \subset E$ um cone convexo fechado $e$ $\xi \in C^{*} \backslash\{0\}$. Então, $\langle\xi, x\rangle>0$ quando $x \in \operatorname{int} C$.

O seguinte teorema prova que a pseudoinvexidade é condição suficiente para que um ponto crítico vetorial seja solução fracamente eficiente. Com efeito:

Teorema 2.2. Se no problema (VOP), f é pseudoinvexa e $\bar{x} \in S$ é um ponto crítico vetorial, então $\bar{x}$ é uma solução fracamente eficiente.

Demostração: De fato, provaremos que se $\bar{x} \in S$ é um ponto crítico vetorial e não é solução fracamente eficiente e obteremos uma contradição. Neste caso, existe $\lambda^{*} \in C^{*} \backslash\{0\}$ tal que

$$
\lambda^{*} \circ D f(\bar{x})=0
$$

e existe $x \in S$ tal que

$$
f(x)-f(\bar{x}) \in-\operatorname{int} C .
$$

Por outro lado, como $f$ é pseudoinvexa, obtemos de (2.2) que

$$
D f(\bar{x}) \eta(x, \bar{x}) \in-\operatorname{int} C,
$$

e (pelo Lema 2.1)

$$
\lambda^{*}(D f(\bar{x}) \eta(x, \bar{x}))=\left[\lambda^{*} \circ D f(\bar{x})\right] \eta(x, \bar{x})<0,
$$

esta última desigualdade contradiz (2.1). Assim, $\bar{x}$ é solução fracamente eficiente de $(\mathrm{VOP})$.

O seguinte resultado é uma generalização do Teorema de Farkas (veja [2]):

Lema 2.2. Sejam $X, Y$ e $V$ espaços vetoriais normados, $A \in \mathcal{L}(X, V)$ e $M \in$ $\mathcal{L}(X, Y)$ dados, $T \subseteq V$ e $Q \subseteq Y$ cones convexos int $Q \neq \phi e b \in-T, s \in-Q$. Supor que o cone $[\bar{A}, b]^{T}\left(T^{*}\right)$ é fraco*-fechado. Então o sistema

$$
\left\{\begin{array}{l}
A x+b \in-T \\
M x+s \in-\text { int } Q
\end{array}\right.
$$

não tem solução se, e somente se, existem $\tau \in Q^{*} \backslash\{0\}, \lambda \in T^{*}$ tais que

$$
\left\{\begin{array}{rl}
\tau M+\lambda A & =0 \\
\langle\lambda, b\rangle & =0 \\
\langle\tau, s\rangle & =0
\end{array} .\right.
$$

O seguinte teorema é o principal resultado desta seção:

Teorema 2.3. A função $f$ em $(V O P)$ é pseudoinvexa em $S$ se, e somente se, cada ponto crítico vetorial é solução fracamente eficiente de (VOP).

Demostração: Segue, do Teorema 2.2, que se $f$ é pseudoinvexa, então cada ponto crítico vetorial é solução fracamente eficiente de (VOP). Agora, suponha que cada ponto crítico vetorial é solução fracamente eficiente de (VOP). Fixemos $\bar{x} \in S$ e consideremos os seguintes sistemas:

$$
f(x)-f(\bar{x}) \in-\operatorname{int} C(x \in S)
$$




$$
D f(\bar{x}) u \in-\operatorname{int} C(u \in E) .
$$

Provaremos que o se o sistema (2.3) tem uma solução, então o sistema ( 2.4) também tem solução. De fato, se (2.3) tem solução, então $\bar{x}$ não é fracamente eficiente e por hipótese, não é um ponto crítico vetorial, i.e., não existe $\lambda^{*} \in C^{*} \backslash\{0\}$ tal que $\lambda^{*} \circ D f(\bar{x})=0$. Fazendo: $A=0 \in \mathcal{L}(E, F), M=D f(\bar{x}) \in \mathcal{L}(E, F), b=0 \in E$ e $s=0 \in F$, obtemos que não existe $\tau \in Q^{*} \backslash\{0\}$ e $\lambda \in Q^{*}$ tal que

$$
\left\{\begin{array}{r}
\tau M+\lambda A=0 \\
\langle\lambda, b\rangle=0 \\
\langle\tau, s\rangle=0
\end{array}\right.
$$

Por outro lado, pelo Lema 2.2, existe $u \in E$ tal que

$$
\left\{\begin{array}{c}
A u+b=0 \in-Q \\
M u+s=D f(\bar{x}) u \in-\operatorname{int} Q
\end{array} .\right.
$$

Em particular, o sistema (2.4) tem solução $u \in E$. Fazendo $\eta(x, \bar{x})=u$, obtemos que $f$ é pseudoinvexa.

\section{Condições Necessárias e Suficientes para Eficiência Fraca para o Problema com Restrições}

Nesta Seção, consideraremos o seguinte problema de otimização vetorial:

$$
\begin{cases}\text { Minimizar } & f(x) \\ \text { sujeito a } & -g(x) \in-K \\ & x \in S \subseteq E\end{cases}
$$

onde $E, F$ e $G$ são espaços de Banach, $C \subset F$ e $K \subset G$ são cones convexos fechados e distintos de $F$ e $G$, respectivamente, int $C \neq \phi, S \subseteq E$ é aberto e não vazio e as funções $f: E \longrightarrow F$ e $g: E \longrightarrow G$ são diferenciáveis em $S$.

Definição 3.1. Dizemos que $(C V O P)$ é $K T$-invexo em $x_{2} \in \mathcal{F}$ se existe uma função vetorial $\eta: S \times S \longrightarrow E$ tal que para cada $x_{1} \in \mathcal{F}$, se verifica:

$$
\left\{\begin{array}{l}
f\left(x_{1}\right)-f\left(x_{2}\right) \in-\operatorname{int} C \Rightarrow D f\left(x_{2}\right) \eta\left(x_{1}, x_{2}\right) \in-\operatorname{int} C \\
-D g\left(x_{2}\right) \eta\left(x_{1}, x_{2}\right) \in K .
\end{array}\right.
$$

e se, o problema é KT-invexo em cada $x \in \mathcal{F}$, dizemos que $(C V O P)$ é KT-invexo.

Para o caso finito-dimensional Osuna-Gómez, Rufián-Lizana e Ruiz-Canales [12] provaram que ser ponto crítico vetorial Kuhn-Tucker é condição necessária e suficiente para a eficiência fraca quando (CVOP) é KT-invexo.

Teorema 3.1. Quando $E=\mathbb{R}^{n}, F=\mathbb{R}^{p}, G=\mathbb{R}^{m}, C=\mathbb{R}_{+}^{p}$ e $K=\mathbb{R}_{+}^{m}$, todo ponto crítico vetorial Kuhn-Tucker é solução fracamente eficiente de (CVOP) se, e somente se, $(C V O P)$ é KT-invexo. 
Agora, enunciaremos e provaremos resultados análogos para o problema infinitodimensional.

Teorema 3.2. (suficiência) Se (CVOP) é um problema KT-invexo, então cada ponto crítico Kuhn-Tucker é solução fracamente eficiente de (CVOP).

Demostração: Assuma que $(C V O P)$ é KT-invexo e seja $\bar{x}$ um ponto crítico Kuhn-Tucker. Neste caso, existem $\lambda^{*} \in C^{*} \backslash\{0\}$ e $\mu^{*} \in K^{*}$ tais que

$$
\begin{gathered}
\lambda^{*} \circ D f(\bar{x})+\mu^{*} \circ D g(\bar{x})=0 \\
\left\langle\mu^{*}, g(\bar{x})\right\rangle=0
\end{gathered},
$$

e, em particular,

$$
\begin{gathered}
\lambda^{*} \circ D f(\bar{x}) \eta(\bar{x}, x)+\mu^{*} \circ D g(\bar{x}) \eta(\bar{x}, x)=0, \forall x \in \mathcal{F}, \\
\left\langle\mu^{*}, g(\bar{x})\right\rangle=0 .
\end{gathered}
$$

Assuma que $\bar{x}$ não é solução fracamente eficiente de (CVOP). Então existe $x \in S$, $g(x) \in-K$ tal que $f(x)-f(\bar{x}) \in-\operatorname{int} C$ e como $\lambda^{*} \in C^{*} \backslash\{0\}$, então pelo Lema 2.1 temos que

$$
\lambda^{*}(D f(\bar{x}) \eta(x, \bar{x}))<0 .
$$

De (3.1) e (3.2), obtemos

$$
\mu^{*}(D g(\bar{x}) \eta(x, \bar{x}))>0 .
$$

Como (CVOP) é KT-invexo, $-D g(\bar{x}) \eta(x, \bar{x}) \in K$ e $\mu^{*} \in K^{*}$, temos que

$$
\mu^{*}(D g(\bar{x}) \eta(x, \bar{x})) \leq 0,
$$

o que contradiz (3.3) e, assim, $\bar{x}$ é solução fracamente eficiente de (CVOP).

Teorema 3.3. (Necessidade) Assuma que $\bar{x}$ é um ponto crítico Kuhn-Tucker de $(C V O P)$ e que o conjunto $[D g(\bar{x}), g(\bar{x})]^{T}\left(K^{*}\right)$ é fraco*-fechado. Então, se cada ponto crítico vetorial Kuhn-Tucker é solução fracamente eficiente de (CVOP), então (CVOP) é KT-invexo.

Demostração: Seja $\bar{x} \in S$ fixo, consideremos os sistemas:

$$
\left\{\begin{array}{l}
D f(\bar{x}) u \in-\operatorname{int} C \\
D g(\bar{x}) u \in-K
\end{array}\right.
$$

$\mathrm{e}$

$$
\left\{\begin{aligned}
f(x)-f(\bar{x}) & \in-\operatorname{int} C \\
g(x) & \in-K .
\end{aligned}\right.
$$

Então provar que (CVOP) é KT-invexo em $\bar{x}$ é equivalente a provar que (3.4) tem solução $u \in E$ quando o sistema (3.5) tem solução $x \in S$ (em tal caso, é suficiente tomar $\eta(x, \bar{x})=u \in E$ ). Assuma que o sistema (3.5) tenha solução. Então, $\bar{x}$ não é solução fracamente eficiente e por hipótese $\bar{x}$ não é ponto crítico vetorial Kuhn-Tucker. Assim, não existem $\tau \in C^{*} \backslash\{0\}$ e $\lambda \in K^{*}$ tais que

$$
\begin{aligned}
\tau M+\lambda A & =0, \\
\langle\lambda, b\rangle & =0, \\
\langle\tau, s\rangle & =0
\end{aligned}
$$


(onde $A=D g(\bar{x}) \in \mathcal{L}(E, G), M=D f(\bar{x}) \in \mathcal{L}(E, F), b=g(\bar{x}) \in-K$ e $s=0 \in$ $-C)$. Segue, do Lema 2.2, que o sistema

$$
\left\{\begin{aligned}
A u+b & \in-K \\
M x+s & \in-\operatorname{int} C
\end{aligned}\right.
$$

tem solução ou, equivalentemente, existe $u \in E$ tal que

$$
\left\{\begin{array}{rl}
D g(\bar{x}) u+g(\bar{x}) & \in-K \\
D f(\bar{x}) u & \in-\operatorname{int} C
\end{array} .\right.
$$

Mas,

$$
D g(\bar{x}) u=[D g(\bar{x}) u+g(\bar{x})]-g(\bar{x}) \in-K-K \subseteq-K
$$

e, assim, o sistema (3.5) tem solução.

Observação: O conjunto $[D g(\bar{x}), g(\bar{x})]^{T}\left(K^{*}\right)$ é fraco* fechado, quando o cone $K$ é poliédrico, qualquer que seja $\bar{x} \in \mathcal{F}$. Veja [4]. Desta maneira,os resultados deste trabalho generalizam os correspondentes obtidos por Osuna-Gómez et al. [12], em que os autores consideram o caso $K=\mathbb{R}_{+}^{p}$.

Observação: Queremos salientar que os resultados obtidos neste trabalho são válidos ainda para espaços normados. Pois, segundo nosso conhecimento o Teorema de Alternativa de Farkas (Lema 2.2 no trabalho) permanece válido (veja [4]), o qual é um dos resultado essenciais na nossa argumentação.

\section{Conclusões}

Este trabalho é uma extensão dos resultados obtidos em [12] para o contexto de otimização entre espaços de Banach, cuja estrutura de dominação é dada por cones. Estes resultados caracterizam os problemas pseudoinvexos (caso irrestrito) e KTinvexos (problemas com restrições) em termos de pontos críticos vetoriais e pontos críticos Kuhn-Tucker, respectivamente.

Agradecimentos $\mathrm{O}$ presente trabalho foi parcialmente financiado pelo Ministerio de Educación de España, através do projeto BFM 2003-06579. A primeira Autora agradece à CAPES (processo número BEX 2444/02-0) pelo financiamento de sua estância na Universidad de Sevilla, durante a qual se realizou este trabalho. Também agradece ao Departamento de Estadística e Investigación Operativa desta universidade pelas facilidades oferecidas durante o referido período de Estágio.

\section{Referências}

[1] A. Avez, "Calcul Differentiel", Mason, Paris, 1983.

[2] B.D. Craven, Lagrangean Conditions and Quasiduality, Bull. Austral. Math. Soc., 16 (1977), 325-339. 
[3] B.D. Craven, "Mathematical Programming and Control Theory", Chapman and Hall: London, 1978.

[4] B.D. Craven, "Control and Optimization", Chapman and Hall, 1995.

[5] R.R. Egudo, M.A. Hanson, Duality with generalized convexity, J. Austral. Math. Soc. Ser. B, 28 (1986), 10-21.

[6] M.A. Hanson, On Sufficiency of Kuhn- Tucker Conditions, J. Math. Anal. and Appl., 30 (1981), 545-550.

[7] V. Jeyakumar, Strong and weak invexity in Mathematical Programming, Collection: Methods of Operations Research, 55 (1980), 109-125.

[8] D. The Luc, "Theory of Vector Optimization", Lect. Notes in Economics and Mathematical Systems, 319, Berlin, Springer-Verlag (1989).

[9] O.L. Mangasarian, "Nonlinear Programming", Mac Graw-Hill: New York, 1969.

[10] D.H. Martin, The Essence of Invexity, J. Optim. Theory Appl., 47 (1985), 65-76.

[11] V. Preda, On duality with generalized convexity, Boll. Un. Mat. Ital. A (7), 5 (1991), 291-305.

[12] R. Osuna-Gómez, A. Rufián-Lizana and P. Ruiz-Canales, Invex Functions and Generalized Convexity in Multiobjective Programming, J. of Optim. Theory Appl., 98 (1998), 651-661.

[13] R. Osuna-Gómez, "Programción con Objetivos Múltiples: Dualidad", Tese de Doutorado, Universidad de Sevilla, España, Sevilla, Espanha, 1996. 